\title{
MACHINE TRANSLATION DEVELOPMENT FOR INDIAN LANGUAGES AND ITS APPROACHES
}

\author{
Amruta Godase ${ }^{1}$ and Sharvari Govilkar ${ }^{2}$ \\ ${ }^{1}$ Department of Information Technology (AI \& Robotics), PIIT, Mumbai University, \\ India \\ ${ }^{2}$ Department of Computer Engineering, PIIT, Mumbai University, India
}

\begin{abstract}
This paper presents a survey of Machine translation system for Indian Regional languages. Machine translation is one of the central areas of Natural language processing (NLP). Machine translation (henceforth referred as MT) is important for breaking the language barrier and facilitating inter-lingual communication. For a multilingual country like INDIA which is largest democratic country in whole world, there is a big requirement of automatic machine translation system. With the advent of Information Technology many documents and web pages are coming up in a local language so there is a large need of good MT systems to address all these issues in order to establish a proper communication between states and union governments to exchange information amongst the people of different states. This paper focuses on different Machine translation projects done in India along with their features and domain.
\end{abstract}

\section{KEYWORDS}

Machine translation, computational linguistics, Indian Languages, Rule-based, Statistical, Empirical MT, Principle-based, Knowledge-based, Hybrid

\section{INTRODUCTION}

Machine translation is one of the central areas of Natural language processing (NLP). Machine translation (MT) is important for breaking the language barrier among the people and for interlingual communication where translation is done from source language to target language. Many researchers, Institutions and organizations in India have started working on MT systems for Indian languages and have gained satisfactory results. The research scenario in India is relatively young and MT gained momentum in India with institutions like IIT Kanpur, IIT Mumbai, IIIT Hyderabad, University of Hyderabad and CDAC Pune play a major role in developing these systems.

This paper is organized into 4 sections. Section 2 gives an introduction of MT \& different approaches to build MT systems, Section 3 discuss major MT systems in India based on language with their features, domain etc. and finally we conclude the paper in the next section. 
International Journal on Natural Language Computing (IJNLC) Vol. 4, No.2,April 2015

\section{MACHINE TRANSLATION}

Like translation done by human, MT does not simply substituting words but the application of complex linguistic knowledge; morphology, grammar, meaning all this things is taken into consideration. Generally, MT is classified into various categories: Direct based, rule-based, corpus based, statistical-based, hybrid-based, example-based, knowledge-based, principle-based, and online interactive based methods. At present, most of the MT related research is based on Rule based approaches because rule based is always extensible and maintainable.

\section{Direct Translation}

Direct Machine Translation is the one of the simplest machine translation approach in which a direct word to word translation is done with the help of a bilingual dictionary.

\section{Rule Based Translation}

A Rule-Based Machine Translation (RBMT) system consists of collection of various rules, called grammar rules, a bilingual lexicon or dictionary, and software programs to process the rules.

\section{Interlingua Based Translation}

In this approach, the translation consists of two stages, where the source Language (SL) is first converted in to the Interlingua (IL) form. The main advantage of Interlingua approach is that the analyzer and parser of SL is independent of the generator for the Target Language (TL) and this requires complete resolution of ambiguity in source language text.

\section{Statistical-based Approach}

Statistical machine translation (SMT) is a data-oriented statistical framework which is based on the knowledge and statistical models which are extracted from bilingual corpora. In this MT, bilingual or multilingual corpora of the languages are required. In SMT, a document is translated according to the probability distribution function which is indicated by $p(e \mid f)$. Finding the best translation is done by picking the highest probability, as shown in Equation 1.

$$
e=\operatorname{argmax} p(e \mid f)=\operatorname{argmax} p(f \mid e) p(e) \ldots \ldots \ldots \ldots(i)
$$

\section{Example-based translation}

Basic idea of this MT is to reuse the examples of already existing translations. An example-based translation is uses a bilingual corpus as its main knowledge base and it is essentially translation by analogy.

\section{Knowledge-Based MT}

Knowledge-Based Machine Translation (KBMT) requires complete understanding of the source text prior to the translation into the target text. KBMT is implemented on the Interlingua 
architecture. KBMT must be supported by world knowledge and by linguistic semantic knowledge about meanings of words and their combinations.

\section{Principle-Based MT}

Principle-Based Machine Translation (PBMT) Systems are based on the Principles \& Parameters Theory of Chomsky's Generative Grammar and which employs parsing method. In this, the parser generates a detailed syntactic structure which contains lexical, phrasal, grammatical information.

\section{Online Interactive Systems}

In this online interactive translation system, the user has authority to give suggestion for the correct translation. This approach is very useful, where the context of a word is not that much clear or unambiguous and where multiple possible meanings for a particular word.

\section{Hybrid-based Translation}

By taking the advantage of statistical MT and rule-based MT methodologies, a new approach was developed, which is termed as "hybrid-based approach". The hybrid approach used in a number of different ways. Translations are performed in the first stage using a rule-based approach which is followed by adjusting or correcting the output using statistical information. Second way in which rules are used to pre-process the input data and for post-process the statistical output of a statistical-based translation system.

\section{LITERATURE SURVEY}

In this section we now look at some major English-Indian language MT project. The parameters we look at are: language pair(s), approaches used for handling problems, year of publication \& application domain of each MT system. The scope of this paper is restricted to Hindi, Punjabi, Bengali, English and Marathi languages as source/target language.

A.Translation system for Hindi language as a Source or Target language

\begin{tabular}{|l|l|l|l|l|l|l|}
\hline $\begin{array}{l}\text { SR } \\
\text { No }\end{array}$ & $\begin{array}{l}\text { Machine } \\
\text { Translation } \\
\text { Systems }\end{array}$ & Year & $\begin{array}{l}\text { Languages } \\
\text { for } \\
\text { translation }\end{array}$ & $\begin{array}{l}\text { Domain / } \\
\text { Application } \\
{[1][47]}\end{array}$ & $\begin{array}{l}\text { Approach } \\
\text { used }\end{array}$ & Observations \\
\hline 1. & $\begin{array}{l}\text { ANUSAARAK MT } \\
\text { If }\end{array}$ & $\begin{array}{l}\text { For translating } \\
\text { children's } \\
\text { stories }\end{array}$ & Direct based & $\begin{array}{l}\text { The focus is on } \\
\text { language access } \\
\text { between Indian } \\
\text { languages. Works } \\
\text { on the principles } \\
\text { of } \\
\text { Paninian } \\
\text { Grammar (PG). }\end{array}$ \\
\hline
\end{tabular}


International Journal on Natural Language Computing (IJNLC) Vol. 4, No.2,April 2015

\begin{tabular}{|c|c|c|c|c|c|c|}
\hline 2. & MANTRA MT [2] & 1997 & $\begin{array}{l}\text { English- } \\
\text { Hindi }\end{array}$ & General & $\begin{array}{l}\text { Transfer } \\
\text { based }\end{array}$ & $\begin{array}{l}\text { It uses TAG \& } \\
\text { XTAG. Uses } \\
\text { tagger and light } \\
\text { dependency } \\
\text { analyzer } \\
\text { performing for } \\
\text { analysis of I/P } \\
\text { English text. It } \\
\text { distributes a load } \\
\text { on man and } \\
\text { machine in equal } \\
\text { way. }\end{array}$ \\
\hline 3. & $\begin{array}{l}\text { MANTRA } \\
\text { RAJYASABHA } \\
{[2][48]}\end{array}$ & 1999 & $\begin{array}{l}\text { English- } \\
\text { Hindi }\end{array}$ & $\begin{array}{l}\text { Office } \\
\text { administration } \\
\text { documents }\end{array}$ & $\begin{array}{l}\text { Transfer } \\
\text { based }\end{array}$ & $\begin{array}{l}\text { System uses TAG } \\
\& \quad \text { LTAG to } \\
\text { represent } \\
\text { grammar. } \\
\text { preserve } \\
\text { formatting the } \\
\text { input of } \\
\text { document. } \\
\text { Currently working } \\
\text { on Hindi to } \\
\text { English and } \\
\text { Hindi- Bengali. }\end{array}$ \\
\hline 4. & ANUBHARTI-I [2] & 2003 & $\begin{array}{l}\text { Hindi- } \\
\text { English }\end{array}$ & General & Hybrid & $\begin{array}{l}\text { Combination of } \\
\text { example based, } \\
\text { corpus based \& } \\
\text { some grammatical } \\
\text { analysis. } \\
\text { reduces It } \\
\text { requirement of } \\
\text { large example } \\
\text { base and it } \\
\text { depends on target } \\
\text { language. }\end{array}$ \\
\hline 5. & $\begin{array}{l}\text { ANUBHARTI-II } \\
\text { [2] }\end{array}$ & 2004 & $\begin{array}{l}\text { Hindi- } \\
\text { English }\end{array}$ & General & Hybrid & $\begin{array}{l}\text { It emulates } \\
\text { human-learning } \\
\text { process for storing } \\
\text { knowledge from } \\
\text { past experience to } \\
\text { use it in future. } \\
\text { Shallow chunker } \\
\text { is used for } \\
\text { fragmentation of } \\
\text { input sentences. }\end{array}$ \\
\hline 6. & $\begin{array}{ll}\text { Hinglish } & \text { MT } \\
\text { System [49] } & \end{array}$ & 2004 & $\begin{array}{l}\text { Hindi- } \\
\text { English }\end{array}$ & General & $\begin{array}{l}\text { Example } \\
\text { based }\end{array}$ & $\begin{array}{l}\text { Based on } \\
\text { Anubharti-II } \\
\text { \&Anglabharti-II. } \\
\text { It produces } \\
\text { satisfactory } \\
\text { results in more } \\
\text { than } 90 \text { cases. It } \\
\text { performs shallow } \\
\text { grammatical } \\
\text { analysis. }\end{array}$ \\
\hline 7. & $\begin{array}{l}\text { An English-Hindi } \\
\text { Translation System }\end{array}$ & 2002 & $\begin{array}{l}\text { English- } \\
\text { Hindi }\end{array}$ & $\begin{array}{l}\text { Weather } \\
\text { narration }\end{array}$ & $\begin{array}{l}\text { Transfer } \\
\text { based }\end{array}$ & $\begin{array}{l}\text { Translation } \\
\text { modules consist }\end{array}$ \\
\hline
\end{tabular}


International Journal on Natural Language Computing (IJNLC) Vol. 4, No.2,April 2015

\begin{tabular}{|c|c|c|c|c|c|c|}
\hline & [3] & & & & & $\begin{array}{l}\text { of preprocessing } \\
\text { and post } \\
\text { processing of } \\
\text { English tree. Also } \\
\text { include generation } \\
\text { of Hindi tree. }\end{array}$ \\
\hline 8. & $\begin{array}{l}\text { UNL-based } \\
\text { English-Hindi MT } \\
\text { System [50] }\end{array}$ & 2001 & $\begin{array}{l}\text { English- } \\
\text { Hindi }\end{array}$ & General & Interlingua & $\begin{array}{l}\text { Based on UNL } \\
\text { grammar. Easy to } \\
\text { add new language } \\
\text { for translation. }\end{array}$ \\
\hline 9. & $\begin{array}{l}\text { MaTra: A Practical } \\
\text { Approach to Fully- } \\
\text { Automatic } \\
\text { Indicative English- } \\
\text { Hindi Machine } \\
\text { Translation [4][51] }\end{array}$ & $\begin{array}{l}2004- \\
2006\end{array}$ & $\begin{array}{l}\text { English- } \\
\text { Hindi }\end{array}$ & $\begin{array}{l}\text { News, annual } \\
\text { report, technical } \\
\text { phrases }\end{array}$ & $\begin{array}{l}\text { Transfer } \\
\text { based }\end{array}$ & $\begin{array}{lr}\text { Based on } & \text { MSIR. } \\
\text { It uses transfer } \\
\text { frame } & \text { like } \\
\text { structure } & \\
\text { representation } \quad \& \\
\text { also } & \text { uses } \\
\text { heuristics } & \text { to } \\
\text { resolved } & \\
\text { ambiguities. } & \\
\end{array}$ \\
\hline 10. & $\begin{array}{l}\text { GB Theory } \text { Based } \\
\text { Hindi To English } \\
\text { Translation System } \\
{[5]}\end{array}$ & 2009 & $\begin{array}{l}\text { Hindi- } \\
\text { English }\end{array}$ & General & $\begin{array}{l}\text { Example } \\
\text { Based }\end{array}$ & $\begin{array}{l}\text { System makes use } \\
\text { of Government } \\
\text { and Binding (GB) } \\
\text { theory for } \\
\text { undertaking } \\
\text { translation. It } \\
\text { consists of a } \\
\text { parsing module } \\
\text { and generating } \\
\text { module. }\end{array}$ \\
\hline 10. & $\begin{array}{l}\text { A Pure EBMT } \\
\text { Approach for } \\
\text { English to Hindi } \\
\text { Sentence } \\
\text { Translation System } \\
\text { [6] }\end{array}$ & 2014 & $\begin{array}{l}\text { English- } \\
\text { Hindi }\end{array}$ & $\begin{array}{l}\text { comparing } \\
\text { sentence to } \\
\text { extract the } \\
\text { translation }\end{array}$ & $\begin{array}{l}\text { Example } \\
\text { based }\end{array}$ & $\begin{array}{l}\text { This system uses } \\
\text { parallel corpora } \\
\text { for translation. It } \\
\text { contains various } \\
\text { modules such as } \\
\text { similarity matrix, } \\
\text { training matrix \& } \\
\text { tagging matrix. }\end{array}$ \\
\hline 11. & $\begin{array}{l}\text { Interlingua based } \\
\text { English-Hindi } \\
\text { Machine } \\
\text { Translation system } \\
\text { and Language } \\
\text { Divergence [7] }\end{array}$ & - & $\begin{array}{l}\text { English- } \\
\text { Hindi }\end{array}$ & General & $\begin{array}{l}\text { Interlingua } \\
\text { and Transfer } \\
\text { based }\end{array}$ & $\begin{array}{l}\text { Here } \\
\text { represents } \\
\text { information } \\
\text { sentence } \\
\text { sentence. Lexical- } \\
\text { semantic } \\
\text { divergence is } \\
\text { handled in L-UW } \\
\text { dictionary. } \\
\text { Syntactic } \\
\text { divergence is } \\
\text { primarily tackled } \\
\text { by analyzers. The } \\
\text { amenability to } \\
\text { generation is } \\
\text { tested through } \\
\text { Marathi } \\
\text { Language. }\end{array}$ \\
\hline
\end{tabular}

B.Translation system for Marathi language as a Source or Target language 
International Journal on Natural Language Computing (IJNLC) Vol. 4, No.2,April 2015

\begin{tabular}{|c|c|c|c|c|c|c|}
\hline $\begin{array}{l}\text { SR } \\
\text { No }\end{array}$ & $\begin{array}{l}\text { Machine } \\
\text { Translation } \\
\text { Systems }\end{array}$ & Year & $\begin{array}{l}\text { Languages } \\
\text { for } \\
\text { translation }\end{array}$ & $\begin{array}{l}\text { Domain / } \\
\text { Application }\end{array}$ & $\begin{array}{l}\text { Approach } \\
\text { used }\end{array}$ & Observations \\
\hline 1. & $\begin{array}{l}\text { English to } \\
\text { Devnagari } \\
\text { Translation for UI } \\
\text { Labels of } \\
\text { Commercial web } \\
\text { based Interactive } \\
\text { Applications [8] }\end{array}$ & 2011 & $\begin{array}{l}\text { English- } \\
\text { Devnagari }\end{array}$ & $\begin{array}{l}\text { Web based } \\
\text { Applications }\end{array}$ & Hybrid & $\begin{array}{l}\text { Used banking } \\
\text { glossary available } \\
\text { on the web site of } \\
\text { RBI to create } \\
\text { multilingual } \\
\text { dictionary. For } \\
\text { lexical analyzer } \\
\text { rules are written by } \\
\text { C languages. Used } \\
\text { Bison tools for } \\
\text { running system. }\end{array}$ \\
\hline 2. & $\begin{array}{l}\text { Extending } \\
\text { capabilities of } \\
\text { English to Marathi } \\
\text { Machine Translator } \\
\text { [9] }\end{array}$ & 2012 & $\begin{array}{l}\text { English- } \\
\text { Marathi }\end{array}$ & General & Rule-based & $\begin{array}{l}\text { Much functionality } \\
\text { can be added for } \\
\text { improving the } \\
\text { performance of } \\
\text { translation. It can } \\
\text { be expanded by } \\
\text { including spelling } \\
\text { and grammatical } \\
\text { checks, sentiment } \\
\text { analysis modules. }\end{array}$ \\
\hline 3. & $\begin{array}{l}\text { Rule based English } \\
\text { to Marathi } \\
\text { translation of } \\
\text { Assertive sentence } \\
{[10]}\end{array}$ & 2013 & $\begin{array}{l}\text { English- } \\
\text { Marathi }\end{array}$ & General & Rule-based & $\begin{array}{l}\text { Database of set of } \\
\text { rules maintained } \\
\text { for mapping. } \\
\text { Bilingual- } \\
\text { Dictionary } \\
\text { database plays very } \\
\text { important role } \\
\text { which is endless. } \\
\text { Open-nlp tools } \\
\text { performing } \\
\text { different processes. }\end{array}$ \\
\hline 4. & $\begin{array}{l}\text { A novel approach } \\
\text { for Interlingual } \\
\text { example-based } \\
\text { translation of } \\
\text { English to Marathi } \\
\text { [11] }\end{array}$ & 2014 & $\begin{array}{l}\text { English- } \\
\text { Marathi }\end{array}$ & General & Hybrid & $\begin{array}{l}\text { System is trained } \\
\text { from bilingual } \\
\text { parallel corpora. } \\
\text { Sentence pairs } \\
\text { contain sentence in } \\
\text { one language with } \\
\text { their translation } \\
\text { into another. Uses } \\
\text { parsing techniques. }\end{array}$ \\
\hline 5. & $\begin{array}{l}\text { Transmuter: An } \\
\text { approach to rule } \\
\text { based English- } \\
\text { Marathi machine } \\
\text { Translation [12] }\end{array}$ & 2014 & $\begin{array}{l}\text { English- } \\
\text { Marathi }\end{array}$ & General & Rule-based & $\begin{array}{l}\text { The focus is on } \\
\text { grammar structure } \\
\text { of target language } \\
\text { that produces } \\
\text { better \& smoother } \\
\text { translation. } \\
\text { Lexicon is built for } \\
\text { morphological \& } \\
\text { semantic } \\
\text { properties. }\end{array}$ \\
\hline
\end{tabular}


International Journal on Natural Language Computing (IJNLC) Vol. 4, No.2,April 2015

C.Translation system for Sanskrit language as a Source or Target language

\begin{tabular}{|c|c|c|c|c|c|c|}
\hline $\begin{array}{l}\text { SR } \\
\text { No }\end{array}$ & $\begin{array}{l}\text { Machine } \\
\text { Translation } \\
\text { Systems }\end{array}$ & Year & $\begin{array}{l}\text { Languages } \\
\text { for } \\
\text { translation }\end{array}$ & $\begin{array}{l}\text { Domain / } \\
\text { Application }\end{array}$ & $\begin{array}{l}\text { Approach } \\
\text { used }\end{array}$ & Observations \\
\hline 1. & $\begin{array}{l}\text { ANN \& Rule based } \\
\text { model for English to } \\
\text { Sanskrit Translation } \\
\text { (EST) [13] }\end{array}$ & 2010 & $\begin{array}{l}\text { English- } \\
\text { Sanskrit }\end{array}$ & General & Rule based & $\begin{array}{l}\text { The system makes } \\
\text { use of feed forward } \\
\text { ANN to make } \\
\text { selection } \\
\text { Sanskrit words and } \\
\text { adjectives from } \\
\text { English to Sanskrit } \\
\text { User Data Vector } \\
\text { (UDV). } \\
\text { system employs } \\
\text { only morphological } \\
\text { markings } \\
\text { identify Subject, } \\
\text { Object, } \\
\text { Preposition etc. }\end{array}$ \\
\hline 2 . & $\begin{array}{l}\text { English To Sanskrit } \\
\text { Machine Translator ( } \\
\text { Lexical parser \& } \\
\text { Semantic Mapper) } \\
{[14]}\end{array}$ & 2010 & $\begin{array}{l}\text { English- } \\
\text { Sanskrit }\end{array}$ & General & Rule based & $\begin{array}{l}\text { This system } \\
\text { consists of } 4 \text { main } \\
\text { modules: Lexical } \\
\text { parser, Semantic } \\
\text { Mapper, Translator } \\
\text { \& Composer. In } \\
\text { this system the } \\
\text { information can be } \\
\text { obtained through } \\
\text { introspection and } \\
\text { analysis. }\end{array}$ \\
\hline 3. & $\begin{array}{l}\text { Etrans- A complete } \\
\text { Framework for } \\
\text { English To Sanskrit } \\
\text { Machine Translation } \\
{[15]}\end{array}$ & 2012 & $\begin{array}{l}\text { English- } \\
\text { Sanskrit }\end{array}$ & General & Rule based & $\begin{array}{l}\text { The translation } \\
\text { model is primarily } \\
\text { based on } \\
\text { formulation } \\
\text { Synchronous } \\
\text { Context } \\
\text { Grammar (SCFG), } \\
\text { a sub set of Context } \\
\text { Free Grammar } \\
\text { (CFG). Top-Down } \\
\text { parsing algorithm is } \\
\text { used for generating } \\
\text { possibilities list. }\end{array}$ \\
\hline 4. & $\begin{array}{l}\text { English to Sanskrit } \\
\text { Translator and } \\
\text { synthesizer [16] }\end{array}$ & 2012 & $\begin{array}{l}\text { English- } \\
\text { Sanskrit }\end{array}$ & General & Rule based & $\begin{array}{lr}\text { In the proposed } \\
\text { algorithm system } \\
\text { integrate traditional } \\
\text { dictionary rule } \\
\text { based approach for } \\
\text { translation. } \\
\text { contains } 2 \text { models } \\
\text { Text to Text } \\
\text { Translator and Text } \\
\text { to } \\
\text { synthesizer module. }\end{array}$ \\
\hline 5. & $\begin{array}{l}\text { English-to-Sanskrit } \\
\text { Machine translation }\end{array}$ & 2012 & $\begin{array}{l}\text { English- } \\
\text { Sanskrit }\end{array}$ & General & $\begin{array}{l}\text { Statistical } \\
\text { based }\end{array}$ & $\begin{array}{l}\text { Described the of a } \\
\text { ubiquitous }\end{array}$ \\
\hline
\end{tabular}


International Journal on Natural Language Computing (IJNLC) Vol. 4, No.2,April 2015

\begin{tabular}{|c|c|c|c|c|c|c|}
\hline & $\begin{array}{l}\text { with ubiquitous } \\
\text { applications [17] }\end{array}$ & & & & & $\begin{array}{lr}\text { translation } & \text { and } \\
\text { language learning } \\
\text { framework, on Mini } \\
2440 \quad \text { SBC, a } \\
\text { growing cellular } \\
\text { phone operating } \\
\text { system } \\
\text { internet with } \\
\text { capabilities. } \\
\text { Translation task is } \\
\text { done by statistical } \\
\text { machine decoder. }\end{array}$ \\
\hline 6. & TranSish [18] & 2014 & $\begin{array}{l}\text { Sanskrit- } \\
\text { English }\end{array}$ & General & Rule-based & $\begin{array}{l}\text { With the help of } \\
\text { Artificial } \\
\text { Intelligence system } \\
\text { provide an interface } \\
\text { which converts } \\
\text { Sanskrit sentences } \\
\text { to English with a } \\
\text { rule based model of } \\
\text { parser and a } \\
\text { semantic Mapper. }\end{array}$ \\
\hline
\end{tabular}

D.Translation system for Bengali language as a Source or Target language

\begin{tabular}{|c|c|c|c|c|c|c|}
\hline $\begin{array}{l}\text { SR } \\
\text { No }\end{array}$ & $\begin{array}{ll}\text { Machine } & \text { Translation } \\
\text { Systems } & \end{array}$ & Year & $\begin{array}{l}\text { Languages } \\
\text { for } \\
\text { translation }\end{array}$ & $\begin{array}{l}\text { Domain / } \\
\text { Application }\end{array}$ & $\begin{array}{l}\text { Approach } \\
\text { used }\end{array}$ & Observations \\
\hline 1. & ANUBAAD [19] & $\begin{array}{l}2000- \\
2004\end{array}$ & $\begin{array}{l}\text { English- } \\
\text { Bengali }\end{array}$ & $\begin{array}{l}\text { News } \\
\text { headlines }\end{array}$ & $\begin{array}{l}\text { Example } \\
\text { based }\end{array}$ & $\begin{array}{l}\text { If the headline is } \\
\text { found in } \\
\text { Generalized } \\
\text { Tagged Example- } \\
\text { base after } \\
\text { synthesis then } \\
\text { Bengali headline } \\
\text { is generated. If the } \\
\text { headline cannot be } \\
\text { translated using } \\
\text { Example-base, } \\
\text { then Generalized } \\
\text { Tagged example- } \\
\text { base or Phrasal } \\
\text { example-base is } \\
\text { going to be used } \\
\text { then after the } \\
\text { heuristic } \\
\text { translation } \\
\text { strategy is used. }\end{array}$ \\
\hline 2. & $\begin{array}{l}\text { VAASAANUBAADA } \\
{[20]}\end{array}$ & 2002 & $\begin{array}{l}\text { Bengali- } \\
\text { Assamese }\end{array}$ & News text & $\begin{array}{l}\text { Example } \\
\text { based }\end{array}$ & $\begin{array}{l}\text { Bilingual corpus } \\
\text { is constructed and } \\
\text { aligned manually. } \\
\text { Longer sentences } \\
\text { are fragmented at } \\
\text { punctuation to } \\
\text { obtain better } \\
\text { quality translation. }\end{array}$ \\
\hline
\end{tabular}


International Journal on Natural Language Computing (IJNLC) Vol. 4, No.2,April 2015

\begin{tabular}{|c|c|c|c|c|c|c|}
\hline 3. & $\begin{array}{l}\text { Exploiting Alignment } \\
\text { Techniques in MATREX: } \\
\text { the DCU Machine } \\
\text { Translation System [21] }\end{array}$ & 2008 & $\begin{array}{l}\text { English- } \\
\text { Bengali }\end{array}$ & $\begin{array}{l}\text { Conference } \\
\text { papers }\end{array}$ & $\begin{array}{l}\text { Example } \\
\text { based }\end{array}$ & $\begin{array}{l}\text { Makes use of } \\
\text { marker-based } \\
\text { chunking, } \\
\text { psycholinguistic } \\
\text { constraint which } \\
\text { signifies context. } \\
\text { System uses an } \\
\text { "edit distance } \\
\text { style" dynamic } \\
\text { programming } \\
\text { alignment } \\
\text { algorithm for } \\
\text { alignment } \\
\text { purpose. }\end{array}$ \\
\hline 4. & $\begin{array}{l}\text { A hybrid approach for } \\
\text { Bengali to Hindi MT } \\
\text { System [22] }\end{array}$ & 2009 & $\begin{array}{l}\text { Bengali- } \\
\text { Hindi }\end{array}$ & General & Hybrid & $\begin{array}{l}\text { Multi-engine } \\
\text { Machine } \\
\text { Translation } \\
\text { approach which } \\
\text { Uses } \\
\text { integration an } \\
\text { SMT with a } \\
\text { lexical transfer } \\
\text { based system } \\
\text { (RBMT). The } \\
\text { BLEU scores of } \\
\text { SMT and lexical } \\
\text { transfer based } \\
\text { system are } 0.1745 \\
\text { and .0424 } \\
\text { respectively. The } \\
\text { BLEU score of } \\
\text { hybrid system is } \\
\text { better which is } \\
0.2275\end{array}$ \\
\hline 5. & $\begin{array}{l}\text { Lattice Based Lexical } \\
\text { Transfer in Bengali Hindi } \\
\text { MT Framework [23] }\end{array}$ & 2011 & $\begin{array}{l}\text { Bengali- } \\
\text { Hindi }\end{array}$ & General & Hybrid & $\begin{array}{lr}\begin{array}{l}\text { Lattice } \\
\text { combined }\end{array} & \begin{array}{r}\text { based } \\
\text { with }\end{array} \\
\text { transfer } & \text { based } \\
\text { Uses a } & \text { lattice- } \\
\text { based } & \text { data } \\
\text { structure } & \text { which is } \\
\text { a } & \text { weighted } \\
\text { directed } & \text { acyclic } \\
\text { graph. }\end{array}$ \\
\hline 6. & $\begin{array}{l}\text { English To Bengali } \\
\text { Machine Translation } \\
\text { Using Context Free } \\
\text { Grammars [24] }\end{array}$ & 2013 & $\begin{array}{l}\text { English- } \\
\text { Bengali }\end{array}$ & General & Rule based & $\begin{array}{l}\text { Proposed system } \\
\text { uses sentence } \\
\text { construction rules } \\
\text { in the form of } \\
\text { CGF grammars. } \\
\text { English-Bengali } \\
\text { dictionary has } \\
\text { been designed } \\
\text { which support } \\
\text { efficient } \\
\text { translation. }\end{array}$ \\
\hline
\end{tabular}

E.Translation system for Punjabi language as a Source or Target language 
International Journal on Natural Language Computing (IJNLC) Vol. 4, No.2,April 2015

\begin{tabular}{|c|c|c|c|c|c|c|}
\hline $\begin{array}{l}\text { SR } \\
\text { No }\end{array}$ & $\begin{array}{l}\text { Machine } \\
\text { Translation } \\
\text { Systems }\end{array}$ & Year & $\begin{array}{l}\text { Languages } \\
\text { for } \\
\text { translation }\end{array}$ & $\begin{array}{l}\text { Domain / } \\
\text { Application }\end{array}$ & $\begin{array}{l}\text { Approach } \\
\text { used }\end{array}$ & Observations \\
\hline 1. & $\begin{array}{l}\text { A Web } r \text { Based } \\
\text { English to Punjabi } \\
\text { MT System for } \\
\text { News Headlines [25] }\end{array}$ & 2013 & $\begin{array}{l}\text { English- } \\
\text { Punjabi }\end{array}$ & $\begin{array}{l}\text { News } \\
\text { Headline }\end{array}$ & $\begin{array}{l}\text { Hybrid } \\
\text { approach }\end{array}$ & $\begin{array}{l}\text { Using Rule Based } \\
\text { Approach, system } \\
\text { parses the source } \\
\text { text and produces an } \\
\text { intermediate } \\
\text { representation. The } \\
\text { accuracy of the } \\
\text { system is near about } \\
81.67 \% \text {. }\end{array}$ \\
\hline 2. & $\begin{array}{l}\text { Statistical Machine } \\
\text { Translation } \\
\text { Punjabi to English } \\
\text { Transliteration } \\
\text { systems for proper } \\
\text { noun [26] }\end{array}$ & 2013 & $\begin{array}{l}\text { Punjabi- } \\
\text { English }\end{array}$ & $\begin{array}{l}\text { Government } \\
\text { documents }\end{array}$ & $\begin{array}{l}\text { Statistical } \\
\text { based }\end{array}$ & $\begin{array}{l}\text { The proposed } \\
\text { system is divided to } \\
\text { two parts - learning } \\
\text { and transliteration. } \\
\text { System Accuracy is } \\
\text { depends on data } \\
\text { stored into the } \\
\text { database and overall } \\
\text { accuracy of the } \\
\text { system is measured } \\
\text { upto } 97 \% \text {. }\end{array}$ \\
\hline 3. & $\begin{array}{l}\text { Rule Based Machine } \\
\text { Translation of Noun } \\
\text { Phrases from Punjabi } \\
\text { to English [27] }\end{array}$ & 2010 & $\begin{array}{l}\text { Punjabi- } \\
\text { English }\end{array}$ & $\begin{array}{l}\text { To convert a } \\
\text { noun phrase }\end{array}$ & Transfer & $\begin{array}{l}\text { The system has } \\
\text { analysis, translation } \\
\text { and synthesis } \\
\text { component. } \\
\text { Punjabi morph } \\
\text { analyzer is being } \\
\text { used for analyzing } \\
\text { the } \\
\text { grammatical exact } \\
\text { structure of the } \\
\text { word. Overall } \\
\text { accuracy } \\
\text { translation } \\
85.33 \% \text {. }\end{array}$ \\
\hline 4. & $\begin{array}{lr}\text { Web Based Hindi to } \\
\text { Punjabi } & \text { Machine } \\
\text { Translation } & \text { System } \\
{[28]} & \end{array}$ & 2010 & $\begin{array}{l}\text { Hindi- } \\
\text { Punjabi }\end{array}$ & News paper & Direct based & $\begin{array}{l}\text { The present system } \\
\text { is translating any } \\
\text { complex sentence. } \\
\text { The System } \\
\text { accuracy is } 95 \% \text {. }\end{array}$ \\
\hline 5. & $\begin{array}{l}\text { Hindi To Punjabi } \\
\text { Machine Translation } \\
\text { System [29] }\end{array}$ & 2011 & $\begin{array}{l}\text { Hindi- } \\
\text { Punjabi }\end{array}$ & General & $\begin{array}{l}\text { Hybrid } \\
\text { approach }\end{array}$ & $\begin{array}{l}\text { Morphological } \\
\text { analyzer developed } \\
\text { by IIT-H has been } \\
\text { ported which finds } \\
\text { the root word for the } \\
\text { token and its } \\
\text { morphological } \\
\text { features. } \\
\text { accuracy percentage } \\
\text { for the system is } \\
87.60 \% \text {. }\end{array}$ \\
\hline
\end{tabular}

F.Translation system for Urdu language as a Source or Target language 
International Journal on Natural Language Computing (IJNLC) Vol. 4, No.2,April 2015

\begin{tabular}{|c|c|c|c|c|c|c|}
\hline $\begin{array}{l}\text { SR } \\
\text { No }\end{array}$ & $\begin{array}{l}\text { Machine } \\
\text { Translation Systems }\end{array}$ & Year & $\begin{array}{l}\text { Languages } \\
\text { for } \\
\text { translation }\end{array}$ & $\begin{array}{l}\text { Domain / } \\
\text { Application }\end{array}$ & Approach used & Observations \\
\hline 1. & $\begin{array}{l}\text { Rule Based English } \\
\text { to Urdu Machine } \\
\text { Translation [30] }\end{array}$ & -- & English-Urdu & $\begin{array}{lr}\text { Handle } & \text { case } \\
\text { phrases } & \text { and } \\
\text { verb } & \text { post } \\
\text { position } & \end{array}$ & $\begin{array}{l}\text { Transfer } \\
\text { Approach }\end{array}$ & $\begin{array}{l}\text { It describes the } \\
\text { usage of Paninian } \\
\text { theory in Urdu } \\
\text { translation, which } \\
\text { can handle case } \\
\text { phrases and verb } \\
\text { post position very } \\
\text { efficiently. } \\
\text { Moreover, this } \\
\text { framework can } \\
\text { also be used for } \\
\text { other constructs } \\
\text { and and handle } \\
\text { conditional and } \\
\text { comparative } \\
\text { sentences } \\
\text { designing their } \\
\text { TAM. }\end{array}$ \\
\hline 2. & $\begin{array}{lr}\text { Model for } & \text { English- } \\
\text { Urdu } & \text { Statistical } \\
\text { Machine } & \text { Translation } \\
{[31]} & \end{array}$ & 2013 & English-Urdu & General & $\begin{array}{l}\text { Statistical } \\
\text { Approach }\end{array}$ & 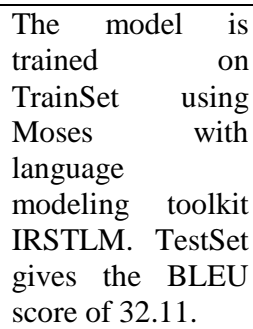 \\
\hline 3. & $\begin{array}{l}\text { Urdu to English } \\
\text { Machine Translation } \\
\text { using Bilingual } \\
\text { Evaluation } \\
\text { Understudy [32] }\end{array}$ & 2013 & Urdu-English & General & Corpus based & 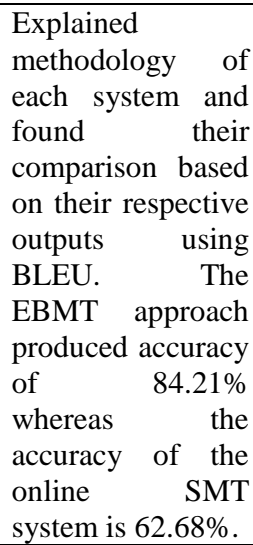 \\
\hline 4. & $\begin{array}{l}\text { Developing } \text { English- } \\
\text { Urdu } \\
\text { Translation Via Hindi } \\
\text { [33] }\end{array}$ & -- & English-Urdu & General & $\begin{array}{l}\text { Interlingua } \\
\text { based Rule- } \\
\text { based approach }\end{array}$ & $\begin{array}{l}\text { The English-Hindi } \\
\text { lexical database is } \\
\text { used for collecting } \\
\text { all possible Hindi } \\
\text { words and also } \\
\text { phrases. Paradigm } \\
\text { file is used for } \\
\text { generating } \\
\text { different } \\
\text { morphological } \\
\text { forms from the } \\
\text { root and entered }\end{array}$ \\
\hline
\end{tabular}


International Journal on Natural Language Computing (IJNLC) Vol. 4, No.2,April 2015

\begin{tabular}{|c|c|c|c|c|c|c|}
\hline & & & & & & $\begin{array}{l}\text { based on } \\
\text { correspondence } \\
\text { with the tags } \\
\text { associated with } \\
\text { Hindi word. These } \\
\text { are then manually } \\
\text { validated. }\end{array}$ \\
\hline 5. & $\begin{array}{l}\text { English to Urdu } \\
\text { Hierarchical Phrase } \\
\text { based statistical MT } \\
{[34]}\end{array}$ & 2013 & English-Urdu & General & $\begin{array}{l}\text { Hierarchical } \\
\text { Phrase based }\end{array}$ & $\begin{array}{l}\text { Uses EMILLE } \\
\text { corpus. Language } \\
\text { model is built on } \\
\text { monolingual Urdu } \\
\text { corpus and it is } \\
\text { implemented as } \\
\text { N-Gram model } \\
\text { using SRILM } \\
\text { toolkit. }\end{array}$ \\
\hline 6. & $\begin{array}{l}\text { VERB TRANSFER } \\
\text { FOR ENGLISH TO } \\
\text { URDU MACHINE } \\
\text { TRANSLATION } \\
\text { (THESIS) }[35]\end{array}$ & 2006 & English-Urdu & $\begin{array}{l}\text { Verb } \\
\text { Transfer }\end{array}$ & Rule-based & $\begin{array}{l}\text { Uses LFG for the } \\
\text { analysis and } \\
\text { generation } \\
\text { modules } \\
\text { machine } \\
\text { translation } \\
\text { systems. Different } \\
\text { senses and sub } \\
\text { categorization } \\
\text { frames were } \\
\text { analyzed. } \\
\text { Ditransitive } \\
\text { Conversion rules } \\
\text { are described to } \\
\text { handle mismatch } \\
\text { of ditransitive } \\
\text { verb analysis in } \\
\text { English and Urdu. }\end{array}$ \\
\hline 7. & $\begin{array}{l}\text { Translation rules and } \\
\text { ANN based model } \\
\text { for English to Urdu } \\
\text { Machine Translation } \\
\text { [36] }\end{array}$ & 2011 & English-Urdu & General & $\begin{array}{l}\text { ANN \& Rule } \\
\text { based }\end{array}$ & $\begin{array}{l}\text { System uses feed- } \\
\text { forward back- } \\
\text { propagation } \\
\text { artificial neural } \\
\text { network for the } \\
\text { selection of Urdu } \\
\text { words/tokens and } \\
\text { translation rules } \\
\text { for grammar } \\
\text { structure } \\
\text { equivalent } \\
\text { English } \\
\text { words/tokens and } \\
\text { grammar structure } \\
\text { rules. The n-gram } \\
\text { blue score } \\
\text { achieved } \\
0.6954 \text {; METEOR } \\
\text { score achieved is } \\
0.8583 \text { and F- } \\
\text { score of } 0.8650 \text {. }\end{array}$ \\
\hline 8. & $\begin{array}{ll}\text { Hindi } & \text { to } \text { Urdu } \\
\text { Machine } & \text { Translation }\end{array}$ & -- & Hindi- Urdu & General & $\begin{array}{l}\text { Character-based } \\
\text { transliteration }\end{array}$ & $\begin{array}{lr}\text { Novel } & \text { approach } \\
\text { proposes } & \text { two }\end{array}$ \\
\hline
\end{tabular}


International Journal on Natural Language Computing (IJNLC) Vol. 4, No.2,April 2015

\begin{tabular}{|c|c|c|c|c|c|c|}
\hline & $\begin{array}{l}\text { Through } \\
\text { Transliteration [37] }\end{array}$ & & & & $\begin{array}{l}\text { model with a } \\
\text { word-based } \\
\text { translation } \\
\text { model. }\end{array}$ & $\begin{array}{l}\text { probabilistic } \\
\text { models, based on } \\
\text { conditional and } \\
\text { joint probability. } \\
\text { Use transliteration } \\
\text { for disambiguation } \\
\text { of Hindi } \\
\text { homonyms which } \\
\text { can be translated } \\
\text { or transliterated or } \\
\text { transliterated } \\
\text { based on different } \\
\text { contexts. }\end{array}$ \\
\hline 9. & $\begin{array}{l}\text { Development of } \\
\text { Parallel Corpus and } \\
\text { English to Urdu } \\
\text { Statistical Machine } \\
\text { Translation [38] }\end{array}$ & 2010 & English-Urdu & General & $\begin{array}{l}\text { Statistical } \\
\text { approach }\end{array}$ & $\begin{array}{l}\text { The whole corpus } \\
\text { was divided into } \\
\text { partitions for the } \\
\text { purpose of cross- } \\
\text { validation. The } \\
\text { alignment of } \\
\text { phrases is } \\
\text { computed based } \\
\text { on word-to-word } \\
\text { alignment. Then } \\
\text { after these } \\
\text { translated phrases } \\
\text { are sequenced } \\
\text { using n-gram } \\
\text { language model. }\end{array}$ \\
\hline
\end{tabular}

\section{G.Translation system for Malayalam language as a Source or Target language}

\begin{tabular}{|c|c|c|c|c|c|c|}
\hline $\begin{array}{l}\text { SR } \\
\text { No }\end{array}$ & $\begin{array}{l}\text { Machine } \\
\text { Translation } \\
\text { Systems }\end{array}$ & Year & $\begin{array}{l}\text { Languages } \\
\text { for } \\
\text { translation }\end{array}$ & $\begin{array}{l}\text { Domain / } \\
\text { Application }\end{array}$ & $\begin{array}{l}\text { Approach } \\
\text { used }\end{array}$ & Observations \\
\hline 1. & $\begin{array}{ll}\text { English } & \text { to } \\
\text { Malayalam } & \\
\text { Translation [39] } & \end{array}$ & 2008 & $\begin{array}{l}\text { English- } \\
\text { Malayalam }\end{array}$ & General & $\begin{array}{l}\text { Statistical } \\
\text { Approach }\end{array}$ & $\begin{array}{l}\text { Monolingual corpus } \\
\text { of Malayalam is } \\
\text { used and bilingual } \\
\text { is used for English } \\
\text { language. The } \\
\text { structural difference } \\
\text { between English } \\
\text { Malayalam pair is } \\
\text { resolved applying } \\
\text { order conversion } \\
\text { rules. }\end{array}$ \\
\hline 2. & $\begin{array}{l}\text { A Hybrid approach } \\
\text { to English to } \\
\text { Malayalam Machine } \\
\text { Translation [40] }\end{array}$ & 2013 & $\begin{array}{l}\text { English- } \\
\text { Malayalam }\end{array}$ & General & $\begin{array}{l}\text { Hybrid } \\
\text { Approach }\end{array}$ & $\begin{array}{lr}\text { A } & \text { statistical } \\
\text { machine } & \text { translator } \\
\text { performs translation } \\
\text { by } & \text { applying } \\
\text { machine learning } \\
\text { techniques on the } \\
\text { corpus. }\end{array}$ \\
\hline 3. & $\begin{array}{lr}\text { Design } & \& \\
\text { Development of a } & \text { Malayalam To } \\
\text { English Translator }\end{array}$ & 2012 & $\begin{array}{l}\text { Malayalam- } \\
\text { English }\end{array}$ & General & $\begin{array}{l}\text { Transfer } \\
\text { Approach }\end{array}$ & $\begin{array}{l}\text { The system consists } \\
\text { of a pre-processor } \\
\text { for splitting the } \\
\text { compound words, a }\end{array}$ \\
\hline
\end{tabular}


International Journal on Natural Language Computing (IJNLC) Vol. 4, No.2,April 2015

\begin{tabular}{|c|c|c|c|c|c|c|}
\hline & [41] & & & & & $\begin{array}{l}\text { morphological } \\
\text { parser for context } \\
\text { disambiguation and } \\
\text { a bilingual } \\
\text { dictionary. The } \\
\text { system is designed } \\
\text { using artificial } \\
\text { intelligence } \\
\text { techniques and can } \\
\text { easily be modified } \\
\text { to build translation } \\
\text { systems for other } \\
\text { language pairs. }\end{array}$ \\
\hline 4. & $\begin{array}{l}\text { Malayalam to } \\
\text { English Machine } \\
\text { Translation [42] }\end{array}$ & 2014 & $\begin{array}{l}\text { Malayalam- } \\
\text { English }\end{array}$ & General & $\begin{array}{l}\text { Example } \\
\text { based }\end{array}$ & $\begin{array}{l}\text { Consist of } 3 \text { phases: } \\
\text { Acquisition, } \\
\text { Matching } \\
\text { Recombination. } \\
\text { The system } \\
\text { searches in the } \\
\text { corpus for each } \\
\text { Malayalam } \\
\text { fragments. About } \\
75 \% \text { of the test } \\
\text { gives good quality } \\
\text { translation. }\end{array}$ \\
\hline
\end{tabular}

H.Translation system for Kannada language as a Source or Target language

\begin{tabular}{|c|c|c|c|c|c|c|}
\hline $\begin{array}{l}\text { SR } \\
\text { No }\end{array}$ & $\begin{array}{ll}\text { Machine } & \text { Translation } \\
\text { Systems } & \end{array}$ & Year & $\begin{array}{l}\text { Languages } \\
\text { for } \\
\text { translation }\end{array}$ & $\begin{array}{l}\text { Domain / } \\
\text { Application }\end{array}$ & $\begin{array}{l}\text { Approach } \\
\text { used }\end{array}$ & Observations \\
\hline 1. & MAT [43] & 2002 & $\begin{array}{l}\text { English- } \\
\text { Kannada }\end{array}$ & $\begin{array}{l}\text { Government } \\
\text { Circulars }\end{array}$ & $\begin{array}{l}\text { Transfer } \\
\text { based }\end{array}$ & $\begin{array}{l}\text { Based on } \\
\text { UCSG. 40-60\% } \\
\text { fully accuracy. } \\
\text { Post editing tool } \\
\text { is provided } \\
\text { which outputs } \\
\text { the number, } \\
\text { type \& inter- } \\
\text { relationships } \\
\text { amongst various } \\
\text { clauses in } \\
\text { sentences. For } \\
\text { each word } \\
\text { suitable target } \\
\text { equivalence is } \\
\text { obtained from } \\
\text { bilingual } \\
\text { dictionary. }\end{array}$ \\
\hline 2. & $\begin{array}{l}\text { A Typical Machine } \\
\text { Translation System for } \\
\text { English-Kannada [44] }\end{array}$ & 2014 & $\begin{array}{l}\text { English- } \\
\text { Kannada }\end{array}$ & General & Rule based & $\begin{array}{l}\text { In this system, } \\
\text { Syntax } \\
\text { reordering } \\
\text { module does } \\
\text { syntactic } \\
\text { differences. } \\
\text { Morphological } \\
\text { generator which }\end{array}$ \\
\hline
\end{tabular}


International Journal on Natural Language Computing (IJNLC) Vol. 4, No.2,April 2015

\begin{tabular}{|l|l|l|l|l|l|}
\hline & & & & & $\begin{array}{l}\text { handle the } \\
\text { complex } \\
\text { morphology of } \\
\text { the target } \\
\text { language. }\end{array}$ \\
\hline
\end{tabular}

I.Machine Translation for English to Indian language

\begin{tabular}{|c|c|c|c|c|c|c|}
\hline $\begin{array}{l}\text { SR } \\
\text { No }\end{array}$ & $\begin{array}{ll}\text { Machine } & \text { Translation } \\
\text { Systems } & \end{array}$ & Year & $\begin{array}{l}\text { Languages } \\
\text { for } \\
\text { translation }\end{array}$ & $\begin{array}{l}\text { Domain / } \\
\text { Application }\end{array}$ & $\begin{array}{l}\text { Approach } \\
\text { used }\end{array}$ & Observations \\
\hline 1. & ANGLABHARTI-I [2] & 2001 & English-IL & Public health & Interlingua & $\begin{array}{l}\text { Creates a PLIL } \\
\text { intermediate } \\
\text { structure. The } \\
\text { effort of PLIL is } \\
70 \% \text { and text } \\
\text { generation is } \\
30 \% \text {. Only with } \\
30 \% \text { new system } \\
\text { can be built. In } \\
\text { this } 90 \% \\
\text { translation work } \\
\text { is done by } \\
\text { machine \& } 10 \% \\
\text { left to the human } \\
\text { post-editing. }\end{array}$ \\
\hline 2. & ANGLABHARTI-II [2] & 2004 & English-IL & General & $\begin{array}{l}\text { Example } \\
\text { based }\end{array}$ & 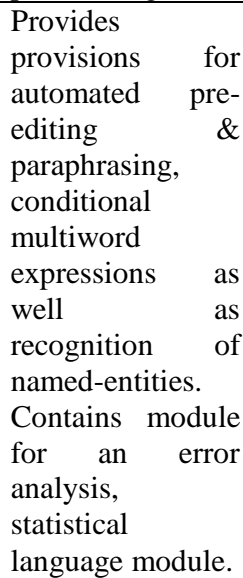 \\
\hline 3. & Shakti [52][53] & 2003 & English-IL & General & $\begin{array}{l}\text { Transfer } \\
\text { based }\end{array}$ & $\begin{array}{l}\text { Linguistic rule } \\
\text { based with } \\
\text { statistical } \\
\text { processing. } \\
\text { Consist of various } \\
\text { modules for } \\
\text { analyzing the } \\
\text { source languages, } \\
\text { performing the } \\
\text { bilingual task and } \\
\text { generating target } \\
\text { Indian language. }\end{array}$ \\
\hline 4. & $\begin{array}{l}\text { Shiva and Shakti MT } \\
\text { System [52][53] }\end{array}$ & 2003 & IL-IL & General & $\begin{array}{l}\text { Example } \\
\text { based }\end{array}$ & $\begin{array}{lr}\text { Easy } & \text { to extend } \\
\text { this } & \text { system for } \\
\text { new } & \text { target }\end{array}$ \\
\hline
\end{tabular}


International Journal on Natural Language Computing (IJNLC) Vol. 4, No.2,April 2015

\begin{tabular}{|c|c|c|c|c|c|c|}
\hline & & & & & & $\begin{array}{lr}\text { language. } & \text { Rules } \\
\text { uses are mostly } \\
\text { linguistic in } \\
\text { nature. Semantic } \\
\text { information is } \\
\text { also used by some } \\
\text { module. }\end{array}$ \\
\hline 5. & AnglaHindi [45] & 2003 & English-Hindi & General & Interlingua & $\begin{array}{l}\text { Pseudo } \\
\text { interlingua based. } \\
\text { Uses all modules } \\
\text { of Anglabharti. } \\
\text { Use an abstracted } \\
\text { example base. } \\
\text { Accuracy is } 90 \% \text {. }\end{array}$ \\
\hline 6. & $\begin{array}{l}\text { "English to Indian } \\
\text { Languages MT System } \\
\text { (E-ILMT)" [46] }\end{array}$ & 2006 & English-IL & $\begin{array}{l}\text { Tourism and } \\
\text { healthcare }\end{array}$ & $\begin{array}{l}\text { Statistical } \\
\text { based }\end{array}$ & 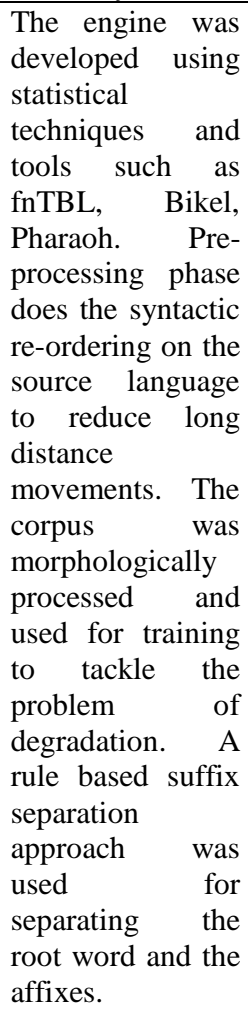 \\
\hline 7. & Google Translator [54] & 2007 & IL-IL & Geneal & $\begin{array}{l}\text { Statistical } \\
\text { based }\end{array}$ & $\begin{array}{l}\text { Google } \\
\text { Translate is a } \\
\text { multilingual } \\
\text { service supports } \\
91 \text { languages. The } \\
\text { system depends } \\
\text { on a solid corpus. } \\
\text { The accuracy of } \\
\text { translation is } \\
\text { better to } \\
\text { understand the } \\
\text { translated text. }\end{array}$ \\
\hline 8. & $\begin{array}{l}\text { ANUVAADAK by IIT- } \\
\text { B [55] }\end{array}$ & & IL-IL & General & $\begin{array}{l}\text { Statistical } \\
\text { Based }\end{array}$ & $\begin{array}{l}\text { The system } \\
\text { enables } \\
\text { translation }\end{array}$ \\
\hline
\end{tabular}


International Journal on Natural Language Computing (IJNLC) Vol. 4, No.2,April 2015

\begin{tabular}{|c|c|c|c|c|c|c|}
\hline & & & & & & $\begin{array}{l}\text { between } 11 \\
\text { different Indian } \\
\text { languages } \\
\text { (including } \\
\text { English), and } \\
\text { provides } \\
\text { transliteration } \\
\text { support for Indic } \\
\text { script input. Users } \\
\text { can post-edit the } \\
\text { translated text } \\
\text { which provides } \\
\text { feedback for } \\
\text { improvement of } \\
\text { the translation } \\
\text { systems }\end{array}$ \\
\hline 9. & Sampark by IIT-H [56] & 2009 & IL-IL & General & $\begin{array}{l}\text { Hybrid } \\
\text { approach }\end{array}$ & $\begin{array}{l}\text { Sampark is a } \\
\text { hybrid system } \\
\text { which is } \\
\text { combination of } \\
\text { traditional rules- } \\
\text { based } \\
\text { algorithms and } \\
\text { dictionaries and } \\
\text { various statistical } \\
\text { machine-learning } \\
\text { techniques. }\end{array}$ \\
\hline
\end{tabular}

\section{CONCLUSION}

The present paper discusses the various language translation systems developed in the India which follows different approaches. The systems are developed for various languages which include various language pairs such as English-Hindi, English-Marathi, English-Sanskrit, English-Malayalam, Malayalam-English, Bengali-Hindi, Bengali-Aasamese, English-Punjabi, Hindi-Punjabi, Punjabi-English/Hindi, English-Kannada, English-Urdu, Urdu-English and IL-IL.

It is concluded that direct approach for Machine Translation is most suitable for closely related languages i.e. the languages with similar structure. The indirect and statistical approach is suitable for languages with different structures.

\section{REFRENCES}

[1] Akshar Bharti, Chaitanya Vineet, Amba P. Kulkarni \& Rajiv Sangal, (1997) ANUSAARAKA: Machine Translation in stages, Vivek, a quarterly in Artificial Intelligence, Vol. 10, No. 3, NCST Mumbai, pp. 22-25.

[2] Sudip Naskar \& Shivaji Bandyopadhyay, (2005) "Use of Machine Translation in India: Current status" AAMT Journal, $\quad$ pp. 25-31

[3] Lata Gore \& Nishigandha Patil, (2002) "English to Hindi - Translation System", In proceedings of Symposium on Translation Support Systems. IIT Kanpur. pp. 178-184 
International Journal on Natural Language Computing (IJNLC) Vol. 4, No.2,April 2015

[4] Ananthakrishnan R, Kavitha M, Jayprasad J Hegde, Chandra Shekhar, Ritesh Shah, Sawani Bade \& Sasikumar M., (2006) "MaTra: A Practical Approach to Fully- Automatic Indicative EnglishHindi Machine Translation", In the proceedings of MSPIL-06

[5] Choudhary,A. Singh, M. (2009) "GB theory based Hindi to english translation system", Computer Science and Information Technology, 2009. ICCSIT 2009. 2nd IEEE International Conference PP. $293-297$

[6] Ruchika A. Sinhal, Kapil O. Gupta (2014) "A Pure EBMT Approach for English to Hindi Sentence Translation System" I.J.Modern Education and Computer Science, 2014, 7, 1-8 Published Online July 2014 in MECS (http://www.mecs-press.org/)

[7] Shachi Dave, Jignashu Parikh And Pushpak Bhattacharyya Department of Computer Science and Engineering, Indian Institute of Technology, Mumbai, India, "Interlingua based English-Hindi Machine Translation system and Language Divergence."

[8] M.L.Dhore \& S.X.Dixit (2011) "English to Devnagari Translation for UI Labels of Commercial web based Interactive Applications" International Journal of Computer Applications ( 0975-8887) Volume 35-No.10, December 2011

[9] Devika Pishartoy, Priya, Sayli Wandkar (2012) "Exteneding capabilities of English to Marathi machine Translator" , International journal of Computer Science Issues, Vol.9, Issues 3, No. 3, May 2012 ISSN (Online): 1694-0814

[10] Abhay A, Anita G, Paurnima T, Prajakta G (2013), "Rule based English to Marathi translation of Assertive sentence", International Journal of Scientific \& Engineering Research, Volume 4, Issues 5, May- 2013 ISSN 2259-5518 pp. 1754-1756

[11] Krushnadeo B, Vinod W, S.V.Phulari, B.S.Kankate (2014), "A novel approach for Interlingual example-based translation of English to Marathi", International Journal of Emerging Technology and Advanced Engineering Website: www.ijetae.com (ISSN 2250-2459, ISO 9001:2008 Certified Journal, Volume 4, Issue 3, March 2014)

[12] G.V.Gajre, G.Kharate, H. Kulkarni (2014), "Transmuter: An approach to Rule-based English to Marathi Machine Translation", International Journal of Computer Applications (0975 - 8887) Volume 98 - No.21, July 2014

[13] Vimal Mishra, R.B.Mishra Research Scholar, Department of Computer Engineering, Institute of Technology, Banaras, Hindu University, (IT-BHU), Varanasi-221005, U.P., India, "ANN and Rule Based Model for English to Sanskrit Machine Translation"

[14] Ms.Vaishali.M.Barkade, Prof. Prakash R. Devale, Dr. Suhas H. Patil, "ENGLISH TO SANSKRIT MACHINE TRANSLATOR LEXICAL PARSER AND SEMANTIC MAPPER", National Conference On "Information and Communication Technology" NCICT-1O

[15] Promila Bahadur ,D.S.Chauhan, , A.K.Jain , Indian Institute of Technology Kanpur, India, “ EtranSA Complete Framework for English To Sanskrit Machine Translation”, IJACSA Special Issue on Selected Papers from International Conference \& Workshop On Emerging Trends In Technology 2012 pp. $52-59$

[16] Sarita G. Rathod, Shanta Sondur, Information Technology Department, VESIT, Mumbai University, Maharashtra, India, "English to Sanskrit Translator and Synthesizer", International Journal of Emerging Technology and Advanced Engineering Website: www.ijetae.com (ISSN 2250-2459, ISO 9001:2008 Certified Journal, Volume 2, Issue 12, December 2012)

[17] Sandeep R. Warhade, Prakash R. Devale ,Suhas H. Patil , “English-to-Sanskrit Statistical Machine Translation with Ubiquitous Application", International Journal of Computer Applications (0975 8887) Volume 51- No.1, August 2012

[18] Pankaj Upadhyay, Umesh Chandra Jaiswal, Kumar Ashish, “ TranSish: Translator from Sanskrit to English-A Rule based Machine Translation", International Journal of Current Engineering and Technology E-ISSN 2277 - 4106, P-ISSN 2347 - 5161 @2014 INPRESSCO®, All Rights Reserved Available at http://inpressco.com/category/ijcet

[19] S. Bandyopadhyay, (2004) "ANUBAAD - The Translator from English to Indian Languages", in proceedings of the VIIth State Science and Technology Congress. Calcutta. India. pp. 43-51 
International Journal on Natural Language Computing (IJNLC) Vol. 4, No.2,April 2015

[20] Kommaluri Vijayanand, Sirajul Islam Choudhury \& Pranab Ratna "VAASAANUBAADA Automatic Machine Translation of Bilingual Bengali-Assamese News Texts", in proceedings of Language Engineering Conference-2002, Hyderabad, India @ IEEE Computer Society.

[21] Yanjun Ma, John Tinsley, Hany Hassan, Jinhua Du \& Andy Way, (2008) "Exploiting Alignment Techniques in MATREX: the DCU Machine Translation System for IWSLT 2008", in proceedings of IWSLT 2008, Hawaii, USA.

[22] Sanjay Chatterji, Devshri Roy, Sudeshna Sarkar, Anupam Basu, 2009, "A Hybrid Approach for Bengali to Hindi Machine Translation" In proceedings of ICON 2009, 7th International Conference on Natural Language Processing. pp. 83-91.

[23] Sanjay Chatterji, Praveen Sonare, Sudeshna Sarkar, and Anupam Basu,2011, "Lattice Based Lexical Transfer in Bengali Hindi Machine Translation Framework", In Proceedings of ICON-2011: 9th International Conference on Natural Language Processing, Macmillan Publishers, India.

[24] Shibli A, Humayun K, Musfique A, K.M.Noman, 2013, "English To Bengali Machine Translation Using Context Free Grammars", International journal of Computer Science Issues, vol.10, Issues 3, No.2, May 2013 ISSN: 1694-0814 pp. 144-153

[25] Harjinder Kaur, Dr. Vijay Laxmi, 2013 "A Web Based English to Punjabi MT System for News Headlines" In International Journal of Advanced Research in Computer Science and Software Engineering 3(6), June - 2013, pp. 1092-1094

[26] Pankaj Kumar and Er.Vinod Kumar, 2013, "Statistical Machine Translation Based Punjabi to English Transliteration System for Proper Nouns", In International Journal of Application or- Innovation in Engineering \& Management (IJAIEM) Volume 2, Issue 8, August 2013 ISSN 2319 - 4847, pp . 318320

[27] Kamaljeet Kaur Batra and G S Lehal, 2010, "Rule Based Machine Translation of Noun Phrases from Punjabi to English", In IJCSI International Journal of Computer Science Issues, Vol. 7, Issue 5, September 2010 ISSN (Online): 1694-0814, pp. 409-413

[28] Vishal Goyal and Gurpreet Singh Lehal , 2010, "Web Based Hindi to Punjabi Machine Translation System", JOURNAL OF EMERGING TECHNOLOGIES IN WEB INTELLIGENCE, VOL. 2, NO. 2, MAY 2010, pp.148-151

[29] Vishal Goyal and Gurpreet Singh Lehal , 2011, "HINDI TO PUNJABI MACHINE TRANSLATION SYSTEM", Proceedings of the ACL-HLT 2011 System Demonstrations, pages 1-6, Portland, Oregon, USA, 21 June 2011. Association for Computational Linguistics

[30] Naila Ata, Bushra Jawaid, Amir Kamran "Rule Based English to Urdu Machine Translation"

[31] Aasim Ali, Arshad, Hussain and Muhammad Kamran Malik, 2013, "Model for English-Urdu Statistical Machine Translation", World Applied Sciences Journal 24 (10): 1362-1367, 2013 ISSN 1818-4952 @ IDOSI Publications, 2013 DOI: 10.5829/idosi.wasj.2013.24.10.760

[32] Asad Habib, Asad Abdul Malik ,Kohat University of Science and Technology, Kohat, Pakistan , 2013, "Urdu to English Machine Translation using Bilingual Evaluation Understudy" International Journal of Computer Applications (0975 - 8887) Volume 82 - No 7, November 2013, pp. 5-12

[33] R. Mahesh K. Sinha Department of Computer Science \& Engineering, Indian Institute of Technology, Kanpur, India, "Developing English-Urdu Machine Translation Via Hindi"

[34] Nadeem khan, Waqas Anwar, Nadir Durrani, 2013, "English to Urdu Hierarchical Phrase based statistical Machine translation"The 4th Workshop on South and Southeast Asian NLP (WSSANLP), International Joint Conference on Natural Language Processing, pages 72-76

[35] Nayyara Karamat December, 2006, "VERB TRANSFER FOR ENGLISH TO URDU MACHINE TRANSLATION (Using Lexical Functional Grammar (LFG)) - MS Thesis" at the National University of Computer \& Emerging Sciences

[36] SHAHNAWAZ, R. B. MISHRA, 2011, "Translation Rules and ANN based model for English to Urdu Machine Translation”, INFOCOMP, v. 10, no. 3, p. 36-47, September of 2011

[37] Nadir Durrani ,Hassan Sajjad ,Alexander Fraser \& Helmut Schmid, Institute for Natural Language Processing University of Stuttgart, "Hindi to Urdu Machine Translation Through Transliteration" 
International Journal on Natural Language Computing (IJNLC) Vol. 4, No.2,April 2015

[38] Aasim Ali, Shahid, Muhammad Malik, 2010, “ Development of Parallel Corpus and English to Urdu Statistical Machine Translation", International Journal of Engineering \& Technology IJET-IJENS Vol:10 No:05, pp.31-33

[39] Mary Priya Sebastian, Sheena Kurian K, G. Santhosh Kumar, 2009 “ English to Malayalam Translation: A Statistical Approach"

[40] Nithya B, Shibily Joseph, 2013 "A Hybrid Approach to English to Malayalam Machine Translation", International Journal of Computer Applications (0975 - 8887) Volume 81 - No.8, November 2013, pp. 11-15

[41] Latha R Nair, David Peter \& Renjith P Ravindran, 2012, "Design and Development of a Malayalam to English Translator-A Transfer Based Approach", International Journal of Computational Linguistics (IJCL), Volume (3) : Issue (1) : 2012

[42] Anju E S, Manoj Kumar K V, 2014, “ Malayalam To English Machine Translation: An EBMT System”, IOSR Journal of Engineering (IOSRJEN) ISSN (e): 2250-3021, ISSN (p): 2278-8719 Vol. 04, Issue 01 (January. 2014), ||V1\| PP 18-23

[43] Murthy. K, (2002) "MAT: A Machine Assisted Translation System", In Proceedings of Symposium on Translation Support System( STRANS-2002), IIT Kanpur. pp. 134-139

[44] Mr. Chethan Chandra S Basavaraddi , Dr. H. L. Shashirekha, 2014, "A Typical Machine Translation System for English to Kannada" , International Journal of Scientific \& Engineering Research, Volume 5, Issue 4, April-2014 ISSN 2229-5518

[45] R.M.K. Sinha \& A. Jain, (2002) "AnglaHindi: An English to Hindi Machine-Aided Translation System", International Conference AMTA(Association of Machine Translation in the Americas)

[46] Vishal Goyal \& Gurpreet Singh Lehal, (2009) "Advances in Machine Translation Systems",National Open Access Journal, Volume 9, ISSN 1930-2940 http://www.languageinindia

[47] http://www.iiit.net/ltrc/Anusaaraka/anu_home.html

[48] http://www.cdac.in/html/aai/mantra.asp

[49] http://www.academia.edu/7986160/Machine_Translation_of_Bilingual_Hindi-English_ Hinglish_Text

[50] http://www.cfilt.iitb.ac.in/machine-translation/ eng-hindi-mt

[51] http://www.ncst.ernet.in/matra/

[52] http://ebmt.serc.iisc.ernet.in/mt/login.html

[53] http://shakti.iiit.net

[54] https://translate.google.co.in

[55] www.cflit.iitb.ac.in/indic-translator/

[56] http://sampark.iiit.ac.in/sampark/

\section{Authors}

Ms. Amruta Godase is Pursuing M.E. in Information Technology with Specialization in Artificial Intelligence \& Robotics from Mumbai University. She received her polytechnic from MSBTE \& B.E from Mumbai University.

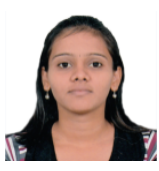

Sharvari Govilkar is Associate professor in Computer Engineering Department, at PIIT, New Panvel, University of Mumbai, India. She has received her M.E in Computer Engineering from University of Mumbai. Currently She is pursuing her $\mathrm{PhD}$ in Information Technology from University of Mumbai. She is having Sixteen years of experience in

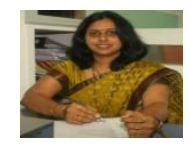
teaching. Her areas of interest are Text Mining, Natural language processing, System programming \& Compiler Design etc. 